\title{
Time-Resolved Dual Frequency Comb Spectroscopy for Broadband Multi-Species Detection in Laser-Induced Plasmas
}

\author{
Caroline Lecaplain ${ }^{1}$, Yu Zhang ${ }^{1,2}$, Reagan R. D. Weeks ${ }^{1}$, Jeremy Yeak ${ }^{3}$, Sivanandan S. Harilal ${ }^{4}$, \\ Mark C. Phillips ${ }^{4}$, R. Jason Jones ${ }^{1}$ \\ 1. College of Optical Sciences, University of Arizona, 1630 E. University Blvd, Tucson, AZ 85721 USA \\ 2. Department of Physics, University of Arizona, 1118 E. Fourth Street, Tucson, AZ 85721, USA \\ 3. Opticslah, Albuquerque, NM USA \\ 4. Pacific Northwest National Laboratory, Richland, WA 99352 USA \\ Email: rjjones@optics.arizona.edu
}

\begin{abstract}
We present the first results using time-resolved broadband dual-comb spectroscopy in a laser-induced plasma. Preliminary results identifying multiple species in a $\mathrm{Nd}$ magnet will be shown.

OCIS codes: (300.6500) Spectroscopy, time-resolved; (280.5395) Plasma diagnostics; (300.1030) Absorption.
\end{abstract}

Laser-induced plasmas provide a novel platform to investigate both fundamental properties of plasmas and plasmaassisted processes like combustion and enable the powerful tools of optical spectroscopy to be applied to the analysis of a broader range of species including solid-state materials and charged ions. It has a wide spectrum of applications, ranging from isotope monitoring to planetary exploration. To obtain the maximum amount of information about the sample composition when optically probing a plasma, it is desirable to utilize a large spectral bandwidth for the identification of multiple ionic, atomic, molecular transitions. Laser-induced breakdown spectroscopy (LIBS), the most common emission technique, is a powerful tool for standoff analysis of solid materials [1]. However, the measurement of a broadband and high-resolution spectrum of multiple atomic species in a single laser ablation shot using LIBS is extremely difficult to achieve. Dual-comb spectroscopy has already been established in the visible to the MIR as a powerful spectroscopic technique. Here, we utilize its ability for rapid detection of transient phenomena to demonstrate broadband, high-resolution, and time-resolved spectroscopy in laser-induced plasmas. Multiple atomic, ionic, and molecular absorption lines can then be tracked simultaneously with high spectral resolution using broadband frequency combs, promising greatly improved plasma characterization and higher detection confidence for species in the plasma. Recently, we reported for the first time, spectroscopy of a highly transient laser-induced plasma using a single laser ablation shot [2]. Here we are extending our technique to perform time-resolved measurements of multiple species following laser ablation of a neodymium magnet.

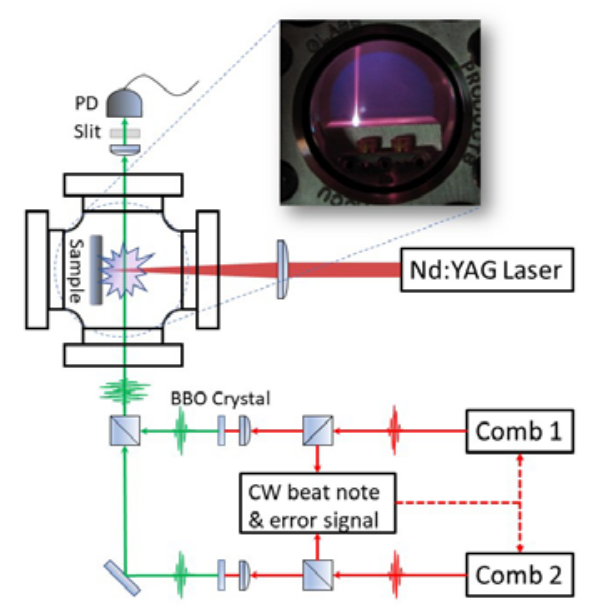

Figure 1. Schematic of the dual-comb laser system to detect broadband multiple species in laser-induced plasmas. Dashed lines indicate electrical phase-lock feedback loop. Inset shows a picture of the laser plasma generated inside the vacuum chamber. PD: photodiode.

A pair of home built Yb-doped mode-locked fiber lasers (Comb-1 and Comb-2) are actively stabilized to a common narrow-linewidth cw laser using optical phase-locked loops. Both fs combs are frequency doubled in BBO crystals. 
A $~ 9$ ns pulse from a Q-switched Nd:YAG laser $(\sim 200 \mathrm{~mJ})$ ablates the $\mathrm{Nd}$ magnet sample inside a vacuum chamber.. The repetition rate difference $\Delta f_{\text {rep }}$ between the lasers was set at around $500 \mathrm{~Hz}$. The ablation laser was triggered at $\sim 10 \mathrm{~Hz}$. The beam from the ablation laser was focused with a $\mathrm{f}=125 \mathrm{~mm}$ lens onto a solid sample placed inside a vacuum chamber with a background pressure of 100 Torr of argon. The dual-comb fs pulses were focused into the vacuum chamber to probe the laser plasma and detected on a photodiode after spectral filtering. The time at which the plasma is probed is critical since the population of various excited states evolves due to the changing plasma temperature. The delay between the ablation pulse and the center burst of the dual-comb interferogram is then controlled such that time-resolved spectra can be obtained. The interferograms were recorded over a time window of 20 ms with a 14 -bit data acquisition card at a sample rate of $125 \mathrm{Ms} / \mathrm{sec}$.

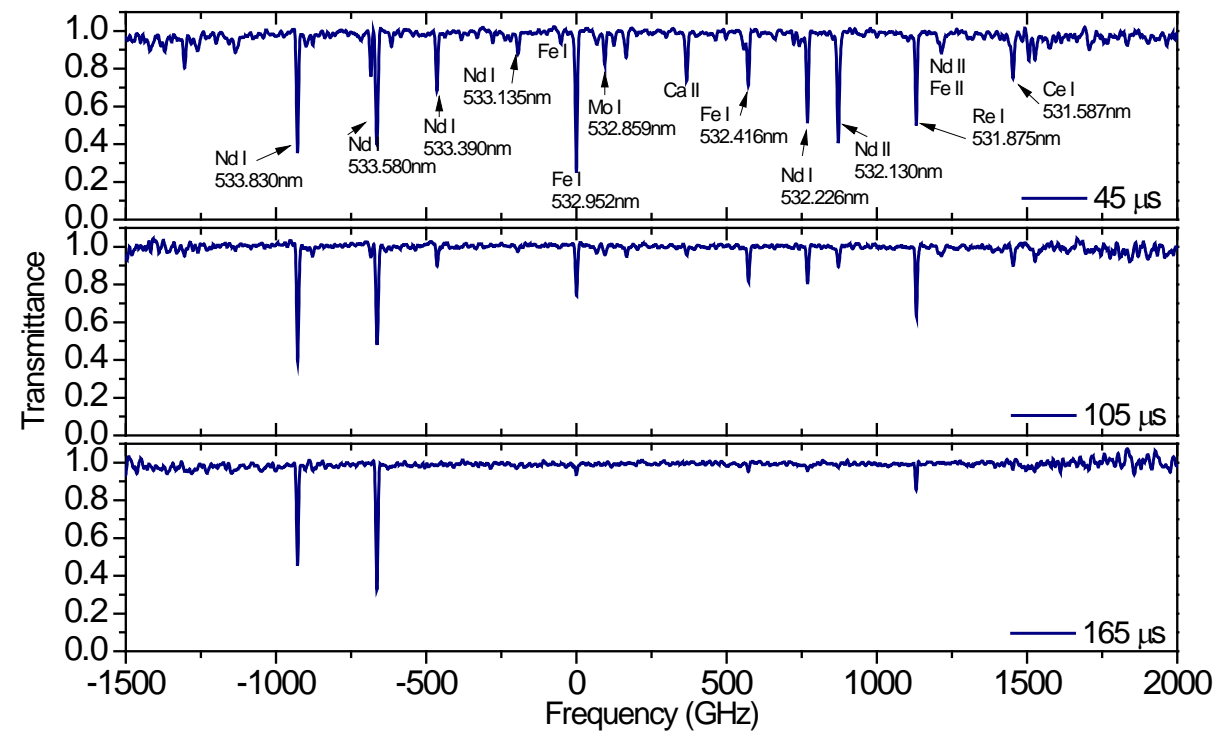

Figure 2. Time-resolved spectra from a Nd magnet sample versus the time delay at which the plasma is probed. Frequency axis shows optical frequency relative to a strong transition of an excited state of Fe I around $533 \mathrm{~nm}$ used as reference. At a time delay of $45 \mu \mathrm{s}$, we detect multiple strong transitions from various species such as Nd I, Fe I, Nd II. The lines tend to disappear with the increase of the time delay as a result of the plasma temperature cooling and the population of the excited states decrease. At a time delay of $165 \mu \mathrm{s}$, only three strong transitions are still observed.

In Fig.2 we present the time-resolved measurements of multiple transitions near 533nm recorded from the $\mathrm{Nd}$ magnet sample while probing the plasma plume at various time delays. For each delay, signal and reference interferograms were recorded simultaneously for 800 laser ablation shots without moving the sample, then Fourier transformed, and averaged in the frequency domain. For short time delays, we observe multiple strong absorption lines. The relative strength of the lines decreases rapidly with time delay as the plasma cools down and the upper states become less populated. Only three strong lines are detected at longer time delays. These preliminary results show that this new absorption spectroscopy approach enables to identify multiple species simultaneously with high resolution and highlight the importance of tracking the time-evolution of the various species within a laser-induced plasma. This work can be extended to study strong ground state transitions of atomic, ionic, and molecular systems in the UV and VUV (e.g. OH, C, N, H, $\mathrm{H}_{2} \ldots$ ) that are important for studies ranging from the characterization of advanced alloys, plasma physics and combustion, to astrophysics and searches for variations of fundamental constants.

Aknowledgements This material is based upon work supported by the Air Force Office of Scientific Research under award number FA9550-15-1-0091 and the Defense Threat Reduction Agency under Grant No. HDTRA 11710030. The Pacific Northwest National Laboratory is operated for the U.S. Department of Energy (DOE) by the Battelle Memorial Institute under Contract No. DE-AC05-76RL01830.

\section{References}

[1] S. S. Harilal, B. E. Brumfield, N. L. LaHaye, K. C. Hartig, and M. C. Phillips "Optical spectroscopy of laser-produced plasmas for standoff isotopic analysis,” Applied Physics Reviews 5, 021301 (2018).

[2] J. Bergevin et. al., "Dual comb spectroscopy of laser-induced plasmas,” Nature Comm. 9, 1273 (2018). 